\title{
Differences in Therapeutic Responses and Factors Affecting Post-Stroke Depression at a Later Stage According to Baseline Depression
}

\author{
Eun-Jae Lee, ${ }^{\mathrm{a}}$ Jong S. Kim, ${ }^{\mathrm{a}}$ Dae-Il Chang, ${ }^{\mathrm{b}}$ Jong-Ho Park, ${ }^{\mathrm{c}}$ Seong Hwan Ahn, ${ }^{\mathrm{d}}$ Jae-Kwan Cha, ${ }^{\mathrm{e}}$ \\ Ji Hoe Heo, ${ }^{\mathrm{f}}$ Sung-Il Sohn, ${ }^{\mathrm{g}}$ Byung-Chul Lee, ${ }^{\mathrm{h}}$ Dong-Eog Kim, ${ }^{\mathrm{i}}$ Hahn Young Kim, ${ }^{\mathrm{j}}$ Seongheon Kim, ${ }^{\mathrm{k}}$ \\ Do-Young Kwon, ${ }^{1}$ Jei Kim, ${ }^{\mathrm{m}}$ Woo-Keun Seo, ${ }^{\mathrm{n}}$ Jun Lee, ${ }^{\mathrm{o}}$ Sang-Won Park, ${ }^{\mathrm{p}}$ Seong-Ho Koh, ${ }^{\mathrm{q}}$ Jin Young Kim, ${ }^{\mathrm{r}}$ \\ Smi Choi-Kwon, ${ }^{,}$Min-Sun Kim, ${ }^{t}$ Ji Sung Lee, ${ }^{\mathrm{u}}$ for the EMOTION Investigators \\ ${ }^{a}$ Department of Neurology, Asan Medical Center, University of Ulsan College of Medicine, Seoul, Korea \\ ${ }^{b}$ Department of Neurology, Kyung Hee University Medical Center, Kyung Hee University School of Medicine, Seoul, Korea \\ 'Department of Neurology, Myongji Hospital, Goyang, Korea \\ 'Department of Neurology, Chosun University Hospital, Chosun University College of Medicine, Gwangju, Korea \\ 'Department of Neurology, Dong-A University Hospital, Dong-A University College of Medicine, Busan, Korea \\ fDepartment of Neurology, Severance Hospital, Yonsei University College of Medicine, Seoul, Korea \\ ${ }^{9}$ Department of Neurology, Keimyung University Dongsan Medical Center, Keimyung University School of Medicine, Daegu, Korea \\ hDepartment of Neurology, Hallym University Sacred Heart Hospital, Hallym University College of Medicine, Anyang, Korea \\ 'Department of Neurology, Dongguk University Ilsan Hospital, Dongguk University College of Medicine, Goyang, Korea \\ 'Department of Neurology, Konkuk University School of Medicine, Seoul, Korea \\ kDepartment of Neurology, Kangwon National University School of Medicine, Chuncheon, Korea \\ 'Department of Neurology, Korea University Asan Hospital, Korea University College of Medicine, Asan, Korea \\ mDepartment of Neurology, Chungnam National University Hospital, Chungnam National University School of Medicine, Daejeon, Korea \\ "Department of Neurology, Samsung Medical Center, Sungkyunkwan University School of Medicine, Seoul, Korea \\ 'Department of Neurology, Yeungnam University Medical Center, Yeungnam University College of Medicine, Daegu, Korea \\ PDepartment of Neurology, Daegu Fatima Hospital, Daegu, Korea \\ "Department of Neurology, Hanyang University Guri Hospital, Hanyang University College of Medicine, Guri, Korea \\ 'Department of Psychiatry, Hyundai Hospital, Eumseong, Korea \\ 'The Research Institute of Nursing Science, Seoul National University College of Nursing, Seoul, Korea \\ ${ }^{t}$ College of Medicine, Michigan State University, East Lansing, MI, USA \\ "Clinical Research Center, Asan Medical Center, University of Ulsan College of Medicine, Seoul, Korea
}

Background and Purpose The pathophysiology of post-stroke depression (PSD) is complex and may differ according to an individual's mood immediately after stroke. Here, we compared the therapeutic response and clinical characteristics of PSD at a later stage between patients with and without depression immediately after stroke.

Methods This study involved a post hoc analysis of data from EMOTION (ClinicalTrials.gov NCT01278498), a placebo-controlled, double-blind trial that examined the efficacy of escitalopram (10 mg/day) on PSD and other emotional disturbances among 478 patients with acute stroke. Participants were classified into the Baseline-Blue (patients with baseline depression at the time of randomization, defined per the Montgomery-Asberg Depression Rating Scale [MADRS] $\geq 8$ ) or the Baseline-Pink groups (patients without baseline depression). We compared the efficacy of escitalopram and predictors of 3-month PSD (MADRS $\geq 8$ ) between these groups.
Correspondence: Jong S. Kim Department of Neurology, Asan Medical Center, University of Ulsan College of Medicine, 88 Olympic-ro 43-gil, Songpa-gu, Seoul 05505, Korea Tel: $+82-2-3010-3440$

Fax: $+82-2-474-4691$

E-mail: jongskim@amc.seoul.kr

Received: November 17, 2017

Revised: May 10, 2018

Accepted: May 11, 2018 
Results There were 203 Baseline-Pink and 275 Baseline-Blue patients. The efficacy of escitalopram in reducing PSD risk was more pronounced in the Baseline-Pink than in the Baseline-Blue group ( $P$ for interaction=0.058). Several risk factors differentially affected PSD development based on the presence of baseline depression ( $P$ for interaction $<0.10$ ). Cognitive dysfunction was an independent predictor of PSD in the Baseline-Blue, but not in the Baseline-Pink group, whereas the non-use of escitalopram and being female were more strongly associated with PSD in the Baseline-Pink group. Conclusions Responses to escitalopram and predictors of PSD 3 months following stroke differed based on the presence of baseline depression. Our data suggest that PSD pathophysiology is heterogeneous; therefore, different therapeutic strategies may be needed to prevent PSD emergence following stroke.

Keywords Depression; Stroke; Escitalopram; Anger; Emotional incontinence

\section{Introduction}

Post-stroke depression (PSD) is common ${ }^{1,2}$ and negatively effects the course of stroke recovery in many patients. ${ }^{3}$ PSD has a dynamic natural course ${ }^{4-8}$ while some patients develop depression immediately following a stroke, others develop it later. This is partly because neurological deficits, which are strongly associated with $\mathrm{PSD}_{1}{ }^{9}$ change dynamically after stroke, especially in the acute/subacute stage. ${ }^{10}$

In the acute stage of stroke, depressive symptoms are fairly common. According to our recent EMOTION (the efficacy of escitalopram on post-stroke emotional disturbances and neurologic dysfunction) trial, ${ }^{11}$ more than half of all acute stroke patients experience depression (per a Montgomery-Asberg Depression Rating Scale [MADRS] score $\geq 8$ ) $^{12}$ immediately following the stroke. Although escitalopram was not effective in reducing the prevalence of moderate to severe PSD (MADRS $\geq 16$ ) at 3 months, it successfully decreased the rate of moderate PSD (MADRS 8-15) at 3 months. Notably, escitalopram was less effective at preventing PSD in the subacute stage in patients with baseline depressive symptoms (the Baseline-Blue group) than in those without (the Baseline-Pink group). These results suggest that the pathogenic mechanism(s) underlying depressive symptoms may differ between these groups. Considering the complex pathophysiology of PSD and the dynamic changes in associated factors such as neurological deficits, we hypothesized that patient response to antidepressants, risk factors, and clinical characteristics of PSD may differ by baseline depression status. In the present study, rather than severe PSD (MADRS $\geq 16$ ), we employed a PSD defined by a MADRS $\geq 8$ as the primary outcome of interest. This score had previously been found to be effectively reduced by escitalopram in a post hoc analysis of our data. ${ }^{11}$

\section{Methods}

\section{Study design and participants}

This is a post hoc analysis of the EMOTION trial." EMOTION was a 24-week, double-blind, placebo-controlled, multicenter trial, which assessed the efficacy of escitalopram on PSD and other emotional disturbances in patients who had experienced acute stroke. In this study, either escitalopram (10 mg/day) or placebo was randomly administered to the patients for 3 months. The placebo was identical to escitalopram in color, shape, and size. Randomization was done in a 1:1 ratio using a web-based system with randomly permuted blocks of four to six and was stratified by center. Patients were followed until 6 months post-stroke (3 months after discontinuation of the study medication). Detailed inclusion and exclusion criteria were described previously." Briefly, patients older than 20 with an acute stroke history (within 21 days of study onset) and a modified Rankin Scale score $\geq 2$ were enrolled by investigators at each participating center. Patients who had a history of depression prior to the index stroke were excluded. Intention-totreat analysis, which included all randomized participants, was used in this post hoc study.

The EMOTION study (ClinicalTrials.gov NCT01278498) was conducted according to Good Clinical Practice guidelines and the Declaration of Helsinki and was approved by the Institutional Review Boards of all participating centers. Informed consent was obtained from all participants.

In this study, we categorized patients into two groups: the Baseline-Blue group, which included patients who experienced depression that developed immediately after the index stroke (MADRS $\geq 8$ ) at the time of randomization, and the BaselinePink group, which included those who did not. 


\section{Assessments}

In the EMOTION study, patients' depressive symptoms were evaluated with MADRS, which consists of a 10-item questionnaire (apparent sadness, reported sadness, inner tension, reduced sleep, reduced appetite, concentration difficulties, lassitude, inability to feel, pessimistic thoughts, and suicidal thoughts) where each item yields a score from 0 to 6 . A higher MADRS score indicates more severe depression. PSD was defined as a MADRS score $\geq 8 .{ }^{12}$ Emotional incontinence was evaluated by Kim's crite$\mathrm{ria}_{1}{ }^{2}$ anger proneness by the modified Spielberger trait anger scale (range from 0 to 40, a higher score indicates higher anger proneness), ${ }^{13}$ neurologic deficits by the National Institute of Health Stroke Scale (NIHSS), ${ }_{1}^{14}$ and cognitive function by the Montreal Cognitive Assessment (MoCA) (score range from 0 to 30; a higher score denotes higher cognitive function)..$^{15}$ Changes in NIHSS scores during the initial month were calculated ("NIHSS score at baseline" - "NIHSS score at 1 month") and used in the analysis of PSD risk factors. In the case of missing data (e.g. patients who dropped out before study termination), we used the most recently available records.

\section{Statistical analyses}

For the univariate analysis, chi-square tests, Fisher's exact tests, Student's t-tests, or Mann-Whitney U tests were used. Baseline characteristics and clinical variables were compared between the Baseline-Pink group and the Baseline-Blue group. In addition, the efficacy of escitalopram 3 months post-stroke was analyzed within each group as were interactions between the effect of escitalopram and the presence of baseline depression. Next, predictors of PSD at 3 months were identified using a multivariable logistic regression model. First, we devised a model including all patients and then compared the odds ratio for each risk factor between the Baseline-Pink and Baseline-Blue groups, also considering interactions with the presence of baseline depression. To explore independent risk factors for PSD at 3 months, we conducted univariate analyses and selected, for a final multivariable model, variables which: (1) were associated with baseline depression, (2) were associated with PSD at 3 months. or (3) demonstrated an interaction with baseline depression for the development of PSD at 3 months. Variables with $P$-values $<0.10$ in any of these analyses were included in multiple logistic regression models. In a multivariable model for the full study cohort, the presence of baseline depression (MADRS $\geq 8$ ) was also included in the analysis to adjust for its effect.

In addition, to examine whether characteristics of PSD are different according to baseline depression, emotional and neurologic disturbances were compared between patients with PSD in the Baseline-Pink and the Baseline-Blue groups. A twotailed t-test value of $P<0.05$ was considered statistically significant. For interactions, $0.05 \leq P<0.10$ was regarded as a significant trend. ${ }^{16}$ All statistical analyses were performed using SPSS version 22.0 (IBM Corp., Armonk, NY, USA) and SAS version 9.4 (SAS Institute Inc., Cary, NC, USA).

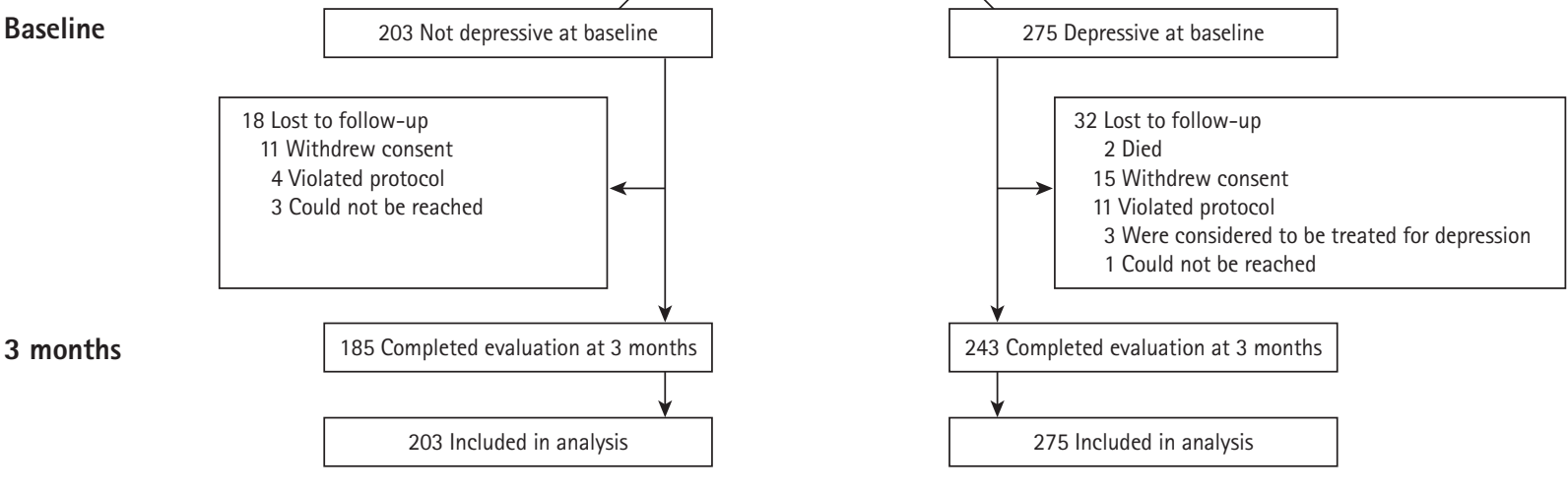

Figure 1. Trial profile. EMOTION, the efficacy of escitalopram on post-stroke emotional disturbances and neurologic dysfunction. 
Table 1. Baseline characteristics of patients with initial depressive symptoms (Baseline-Blue) and those without (Baseline-Pink)

\begin{tabular}{|c|c|c|c|c|}
\hline Characteristic & All patients $(n=478)$ & Baseline-Pink $(n=203)$ & Baseline-Blue $(n=275)$ & $P^{*}$ \\
\hline \multicolumn{5}{|c|}{ Demographic and risk factors } \\
\hline Age (yr) & $64.0 \pm 12.1$ & $62.4 \pm 12.1$ & $65.2 \pm 12.0$ & 0.013 \\
\hline Female sex & $187(39.1)$ & $63(31.0)$ & $124(45.1)$ & 0.002 \\
\hline Hypertension & $360(75.3)$ & 156 (76.8) & $204(74.2)$ & 0.504 \\
\hline Diabetes & $204(42.7)$ & 78 (38.4) & $126(45.8)$ & 0.106 \\
\hline Hyperlipidemia & $235(49.2)$ & $98(48.3)$ & $137(49.8)$ & 0.739 \\
\hline Coronary artery disease & $63(13.2)$ & $24(11.8)$ & $39(14.2)$ & 0.451 \\
\hline Smoking & $230(48.1)$ & $112(55.2)$ & $118(42.9)$ & 0.008 \\
\hline Time-to-randomization & $7(4-10)$ & $7(4-10)$ & $7(5-11)$ & 0.206 \\
\hline Lesion side & & & & 0.357 \\
\hline Right & $239(50.0)$ & $95(46.8)$ & $144(52.4)$ & \\
\hline Left & $208(43.5)$ & $96(47.3)$ & $112(40.7)$ & \\
\hline Both & $31(6.5)$ & $12(5.9)$ & $19(6.9)$ & \\
\hline \multicolumn{5}{|l|}{ Lesion location } \\
\hline Anterior cortex & $126(26.4)$ & $46(22.7)$ & $80(29.1)$ & 0.115 \\
\hline Thalamus & $43(9.0)$ & $21(10.3)$ & $22(8.0)$ & 0.376 \\
\hline Medulla & $39(8.2)$ & $16(7.9)$ & $23(8.4)$ & 0.849 \\
\hline Cerebellum & $37(7.7)$ & $13(6.4)$ & $24(8.7)$ & 0.347 \\
\hline Posterior cortex & $24(5.0)$ & $7(3.4)$ & $17(6.2)$ & 0.176 \\
\hline Subcortex & $226(47.3)$ & $96(47.3)$ & $130(47.3)$ & 0.997 \\
\hline Pons+midbrain & $106(22.2)$ & $44(21.7)$ & $62(22.5)$ & 0.821 \\
\hline \multicolumn{5}{|l|}{ Clinical variables } \\
\hline MADRS & $10.7 \pm 8.2$ & $3.4 \pm 2.4$ & $16.0 \pm 6.7$ & $<0.001$ \\
\hline NIHSS & $4.8 \pm 2.9$ & $4.2 \pm 2.3$ & $5.3 \pm 3.1$ & $<0.001$ \\
\hline Emotional incontinence & $32(6.7)$ & $6(3.0)$ & $26(9.5)$ & 0.005 \\
\hline Anger proneness & $22.8 \pm 5.7$ & $22.6 \pm 5.4$ & $22.9 \pm 5.9$ & 0.528 \\
\hline MoCA & $18.4 \pm 6.9$ & $19.8 \pm 6.5$ & $17.4 \pm 7.1$ & $<0.001$ \\
\hline
\end{tabular}

Values are presented as mean \pm standard deviation, number (\%), or median (interquartile range).

MADRS, Montgomery-Asberg Depression Rating Scale; NIHSS, National Institute of Health Stroke Scale; MoCA, Montreal Cognitive Assessment.

${ }^{*} P$ via two-tailed t-test for Baseline-Pink vs. Baseline-Blue groups.

\section{Results}

Between January 27, 2011 and June 30, 2014, a total of 488 patients were considered for their eligibility, 10 of whom were not included (Figure 1). Given this, 478 patients were included in the present intention-to-treat population and these analyses. Their mean age was $64.0 \pm 12.1$ years, and 187 (39.1\%) were women. The median (interquartile range [IOR]) duration from stroke onset to study randomization was 7 days (IQR, 4 to 10). At baseline, 275 patients (57.5\%) had depression as defined per the MADRS $\geq 8$ (Baseline-Blue), while 203 did not (Baseline-Pink). Compared with the Baseline-Pink, the Baseline-Blue patients were older, more often females, less often smokers, and more likely to have experienced emotional incon- tinence, severe stroke, and cognitive dysfunction (Table 1).

In the Baseline-Pink and Baseline-Blue patients, clinical variables at baseline were well-balanced between the escitalopram and placebo users except for a higher prevalence of emotional incontinence in the placebo arm of the Baseline-Pink group (Supplementary Table 1). Patient responses to escitalopram at 3 months after initiation of therapy were stratified by group (Table 2). In the Baseline-Pink group, patients who were randomized to escitalopram showed significantly lower MADRS scores and less frequent PSD (MADRS $\geq 8$ ) at 3 months. In addition, NIHSS and anger scores tended to be lower among escitalopram users. In the Baseline-Blue group, although the escitalopram group was less likely to have PSD (MADRS $\geq 8$ ) with a marginal statistical significance, escitalopram generally did not 
Table 2. Efficacy of escitalopram according to baseline depressive symptoms

\begin{tabular}{|c|c|c|c|c|c|c|c|}
\hline \multirow[b]{2}{*}{ Variable } & \multicolumn{3}{|c|}{ Baseline-Pink } & \multicolumn{3}{|c|}{ Baseline-Blue } & \multirow{2}{*}{$\begin{array}{c}P \text { for } \\
\text { interaction }^{*}\end{array}$} \\
\hline & $\begin{array}{l}\text { Placebo } \\
(n=109)\end{array}$ & $\begin{array}{l}\text { Escitalopram } \\
\quad(n=94)\end{array}$ & $P$ & $\begin{array}{l}\text { Placebo } \\
(n=128)\end{array}$ & $\begin{array}{c}\text { Escitalopram } \\
\quad(n=147)\end{array}$ & $P$ & \\
\hline \multicolumn{8}{|l|}{ At 3 months } \\
\hline PSD (MADRS score $\geq 8$ ) & $20(18.3)$ & $4(4.3)$ & 0.002 & $81(63.3)$ & $77(52.4)$ & 0.068 & 0.058 \\
\hline MADRS score & $4.1 \pm 4.8$ & $2.4 \pm 2.8$ & 0.002 & $11.1 \pm 8.2$ & $9.6 \pm 7.5$ & 0.121 & 0.840 \\
\hline Emotional incontinence & $10(9.2)$ & $5(5.3)$ & 0.295 & $15(11.7)$ & $10(6.8)$ & 0.157 & 0.987 \\
\hline NIHSS score & $2.5 \pm 2.4$ & $1.9 \pm 1.8$ & 0.062 & $3.2 \pm 2.8$ & $3.4 \pm 3.3$ & 0.579 & 0.134 \\
\hline Anger score & $21.1 \pm 4.9$ & $19.7 \pm 5.1$ & 0.059 & $21.8 \pm 5.8$ & $21.1 \pm 5.6$ & 0.300 & 0.543 \\
\hline MoCA score & $21.9 \pm 6.6$ & $22.1 \pm 6.9$ & 0.806 & $20.0 \pm 6.8$ & $18.8 \pm 7.5$ & 0.155 & 0.258 \\
\hline \multicolumn{8}{|l|}{ At 6 months } \\
\hline PSD (MADRS score $\geq 8$ ) & 25 (22.9) & $6(6.4)$ & 0.001 & $86(67.2)$ & $62(42.2)$ & 0.110 & 0.048 \\
\hline MADRS score & $4.7 \pm 5.4$ & $2.8 \pm 4.0$ & 0.005 & $11.4 \pm 8.0$ & $10.4 \pm 7.5$ & 0.293 & 0.474 \\
\hline Emotional incontinence & $11(10.1)$ & $9(9.6)$ & 0.902 & $16(12.5)$ & $12(8.2)$ & 0.236 & 0.503 \\
\hline NIHSS score & $2.2 \pm 2.4$ & $1.7 \pm 1.8$ & 0.071 & $3.0 \pm 3.0$ & $3.2 \pm 3.4$ & 0.641 & 0.165 \\
\hline Anger score & $20.7 \pm 5.2$ & $20.0 \pm 5.5$ & 0.379 & $21.4 \pm 5.8$ & $21.3 \pm 6.1$ & 0.928 & 0.573 \\
\hline MoCA score & $22.2 \pm 6.8$ & $22.2 \pm 7.1$ & 0.969 & $20.0 \pm 7.1$ & $19.1 \pm 7.6$ & 0.278 & 0.451 \\
\hline
\end{tabular}

Values are presented as number (\%) or mean \pm standard deviation.

PSD, post-stroke depression; MADRS, Montgomery-Asberg Depression Rating Scale; NIHSS, National Institute of Health Stroke Scale; MoCA, Montreal Cognitive Assessment.

${ }^{*}$ P for interaction according to the presence of baseline depressive symptoms (MADRS $\geq 8$ ).

improve emotional and neurological disturbances to a significant degree. The favorable effect of escitalopram on patients' depressive symptoms in the Baseline-Pink group was persistent up to 6 months after the index stroke (i.e., 3 months after medication cessation). Of note, there was a trend interaction between baseline depression and the effect of escitalopram on PSD prevention at 3 months ( $P$ for interaction $=0.058$ ), which was significant at 6 months ( $P$ for interaction $=0.048$ ).

Next, we analyzed independent predictors of 3-month PSD and explored whether they would be different based on the presence of baseline depression. Candidate variables were selected as those with a $P<0.10$. Differences between the Baseline-Pink and the Baseline-Blue groups were significant in terms of age, female percentage, smoking rates, NIHSS, emotional incontinence rates, and MoCA scores (Table 1). PSD at 3 months was associated with old age, being female, a diagnosis of diabetes or hyperlipidemia, non-use of escitalopram, baseline NIHSS, anger levels, and MoCA scores (Table 3). The variables showing an interaction with baseline depression for 3-month PSD were non-use of escitalopram, anger scores, MoCA scores, and changes in NIHSS score (0 to 1 month) (Supplementary Table 2). The presence of baseline depression (MADRS $\geq 8$ ) was also adjusted via a multivariable model for all patients.

In the final model (Table 4) including all patients, the presence of baseline depression (MADRS $\geq 8$ ) was most strongly as- sociated with PSD at 3 months. In addition, higher baseline NIHSS scores and anger scores, cognitive dysfunction, and nonuse of escitalopram were identified as independent predictors of the development of PSD at 3 months. Changes in NIHSS scores were marginally significant. We then assessed whether the effect of each risk factor on PSD at 3 months would differ according to the presence of baseline depression. In the Baseline-Pink group, female sex, baseline NIHSS score, and non-use of escitalopram significantly increased the odds of PSD at 3 months, while in the Baseline-Blue group, hyperlipidemia, higher anger scores, cognitive dysfunction (MoCA scores), changes in NIHSS scores, and non-use of escitalopram were significantly associated with PSD at 3 months. Among these variables, cognitive dysfunction, being female, and non-use of escitalopram demonstrated statistically significant interactions or trends towards interactions with baseline depression ( $P$ for interaction $<0.10$ ). Although non-use of escitalopram was an independent predictor of 3-month PSD in both the BaselinePink and Baseline-Blue groups, the PSD odds ratio was substantially greater in the Baseline-Pink group than in the Baseline-Blue group.

Figure 2 illustrates changes in MADRS scores throughout the study period. In the Baseline-Pink group, placebo and escitalopram users displayed different patterns; patients randomized to the placebo treatment showed a gradual increase in MADRS 
Table 3. Risk factors for post-stroke depression (MADRS $\geq 8$ ) at 3 months in all the patients

\begin{tabular}{|c|c|c|c|}
\hline \multirow{2}{*}{ Variable } & \multicolumn{2}{|c|}{ PSD at 3 months } & \multirow{2}{*}{$P$} \\
\hline & Without $(n=296)$ & With $(n=182)$ & \\
\hline \multicolumn{4}{|l|}{ Demographic and risk factors } \\
\hline Age (yr) & $62.5 \pm 11.8$ & $66.4 \pm 12.4$ & 0.001 \\
\hline Female sex & $107(36.1)$ & $80(44.0)$ & 0.089 \\
\hline Hypertension & 75 (25.3) & $43(23.6)$ & 0.673 \\
\hline Diabetes & $116(39.2)$ & $88(48.4)$ & 0.049 \\
\hline Hyperlipidemia & $136(45.9)$ & $99(54.4)$ & 0.073 \\
\hline Coronary artery disease & $38(12.8)$ & $25(13.7)$ & 0.778 \\
\hline Smoking & $145(49.0)$ & $85(46.7)$ & 0.628 \\
\hline Lesion side & & & 0.131 \\
\hline Right & $144(48.6)$ & $95(52.2)$ & \\
\hline Left & $137(46.3)$ & $71(39.0)$ & \\
\hline Both & $15(5.1)$ & $16(8.8)$ & \\
\hline \multicolumn{4}{|l|}{ Lesion location } \\
\hline Anterior cortex & $72(24.3)$ & $54(29.7)$ & 0.198 \\
\hline Thalamus & $29(9.8)$ & $14(7.7)$ & 0.435 \\
\hline Medulla & $25(8.4)$ & $14(7.7)$ & 0.770 \\
\hline Cerebellum & $20(6.8)$ & $17(9.3)$ & 0.305 \\
\hline Posterior cortex & $13(4.4)$ & $11(6.0)$ & 0.422 \\
\hline Subcortex & $135(45.6)$ & $91(50.0)$ & 0.350 \\
\hline Pons+Midbrain & $61(20.6)$ & $45(24.7)$ & 0.293 \\
\hline \multicolumn{4}{|l|}{ Clinical variables at baseline } \\
\hline Non-use of escitalopram (10 mg/day) & $160(54.1)$ & $81(44.5)$ & 0.043 \\
\hline MADRS & $7.1 \pm 5.9$ & $16.4 \pm 8.2$ & $<0.001$ \\
\hline NIHSS score & $4.4 \pm 2.6$ & $5.5 \pm 3.1$ & $<0.001$ \\
\hline Changes in NIHSS (0-1 mo) & $1.5 \pm 1.6$ & $1.3 \pm 1.5$ & 0.147 \\
\hline Emotional incontinence & $21(7.1)$ & $11(6.0)$ & 0.655 \\
\hline Anger score & $22.1 \pm 5.8$ & $23.9 \pm 5.2$ & $<0.001$ \\
\hline MoCA score & $19.7 \pm 6.3$ & $16.3 \pm 7.3$ & $<0.001$ \\
\hline
\end{tabular}

Values are presented as mean \pm standard deviation or number (\%).

MADRS, Montgomery-Asberg Depression Rating Scale; PSD, post-stroke depression; NIHSS, National Institute of Health Stroke Scale; MoCA, Montreal Cognitive Assessment.

scores over time while those randomized to escitalopram demonstrated an improvement; a significant difference in MADRS scores between treatment groups was demonstrated at 3 months and maintained at 6 months. In the Baseline-Blue group, however, both placebo and escitalopram users showed a uniform decrease in MADRS scores for the first 3 months without significant difference between treatment groups. Improvements were most prominent during the first month and gradually diminished thereafter.

Lastly, clinical characteristics were compared between PSD (at 3 months) patients in the Baseline-Pink and Baseline-Blue groups (Table 5). At baseline, all clinical variables, except for depressive symptoms and cognitive dysfunction, were compa- rable. At 3 months, depressive symptoms (MADRS score) and cognitive dysfunction (MoCA score) remained diminished while emotional incontinence was more prevalent in PSD patients in the Baseline-Pink group.

\section{Discussion}

We examined various clinical characteristics and factors associated with PSD according to the depression levels immediately following a stroke. As the data were obtained as part of a randomized controlled study, risk factors and clinical variables were prospectively collected and well balanced between the two treatment arms. We found that responses to escitalopram 
Table 4. Independent predictors of post-stroke depression (MADRS $\geq 8$ ) at 3 months

\begin{tabular}{|c|c|c|c|c|c|c|c|}
\hline \multirow{2}{*}{ Variable } & \multicolumn{2}{|c|}{ All patients } & \multicolumn{2}{|c|}{ Baseline-Pink } & \multicolumn{2}{|c|}{ Baseline-Blue } & \multirow{2}{*}{$\begin{array}{c}P \text { for } \\
\text { interaction }\end{array}$} \\
\hline & OR $(95 \% \mathrm{Cl})$ & $P$ & OR $(95 \% \mathrm{Cl})$ & $P$ & OR $(95 \% \mathrm{Cl})$ & $P$ & \\
\hline Non-use of escitalopram (10 mg/day) & $2.28(1.44-3.64)$ & $<0.001$ & $6.69(1.96-22.86)$ & 0.002 & $1.93(1.12-3.33)$ & 0.018 & 0.070 \\
\hline Age $(/ 10 \mathrm{yr})$ & $1.01(0.99-1.03)$ & 0.494 & $1.26(0.79-2.00)$ & 0.333 & $1.04(0.80-1.36)$ & 0.769 & 0.488 \\
\hline Female sex & $1.36(0.70-2.62)$ & 0.363 & $4.46(1.04-19.16)$ & 0.044 & $1.05(0.48-2.27)$ & 0.911 & 0.085 \\
\hline Diabetes & $1.14(0.72-1.80)$ & 0.569 & $0.59(0.22-1.64)$ & 0.317 & $1.26(0.73-2.17)$ & 0.407 & 0.203 \\
\hline Hyperlipidemia & $1.48(0.94-2.33)$ & 0.088 & $0.97(0.37-2.54)$ & 0.945 & $1.78(1.04-3.05)$ & 0.035 & 0.277 \\
\hline Smoking & $1.70(0.89-3.26)$ & 0.108 & $2.56(0.69-9.52)$ & 0.161 & $1.39(0.64-3.05)$ & 0.407 & 0.436 \\
\hline Emotional incontinence & $0.48(0.21-1.11)$ & 0.085 & $0.52(0.05-5.50)$ & 0.589 & $0.46(0.18-1.15)$ & 0.096 & 0.919 \\
\hline Baseline NIHSS score & $1.10(1.01-1.20)$ & 0.025 & $1.24(1.01-1.54)$ & 0.042 & $1.07(0.97-1.17)$ & 0.201 & 0.190 \\
\hline Anger score & $1.06(1.01-1.10)$ & 0.010 & $1.01(0.92-1.11)$ & 0.815 & $1.08(1.02-1.13)$ & 0.004 & 0.268 \\
\hline MoCA score & $0.95(0.92-0.99)$ & 0.021 & $1.07(0.98-1.18)$ & 0.125 & $0.92(0.88-0.97)$ & 0.001 & 0.005 \\
\hline Changes in NIHSS & $0.88(0.75-1.03)$ & 0.104 & $1.04(0.74-1.47)$ & 0.816 & $0.83(0.68-0.99)$ & 0.046 & 0.248 \\
\hline Baseline depression (MADRS $\geq 8$ ) & $11.23(6.58-19.17)$ & $<0.001$ & - & & - & & \\
\hline
\end{tabular}

MADRS, Montgomery-Asberg Depression Rating Scale; OR, odds ratio; Cl, confidence interval; NIHSS, National Institute of Health Stroke Scale; MoCA, Montreal Cognitive Assessment.

${ }^{*}$ P for interaction according to the presence of baseline depressive symptoms (MADRS $\geq 8$ ).

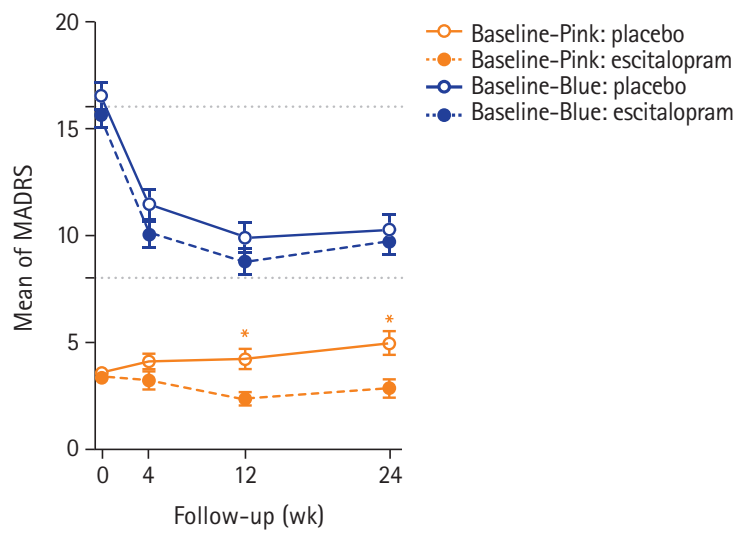

Figure 2. Changes in Montgomery-Asberg Depression Rating Scale (MADRS) scores during the study period. ${ }^{*} P<0.001$.

and predictors for PSD at 3 months differed between the Baseline-Blue and Baseline-Pink groups.

The efficacy of escitalopram was more marked in the BaselinePink than in the Baseline-Blue group (Table 2). PSD risk and MADRS scores at 3 months were significantly lower in the escitalopram users than in the placebo users only in the BaselinePink group. Of note, in reducing the PSD at 3 months, there was a trend interaction between baseline depression and escitalopram use. Moreover, non-use of escitalopram was a more important predictor of PSD at 3 months in the Baseline-Pink than in the Baseline-Blue groups (Table 4). Perhaps, in the Baseline-Blue group, factors such as neurologic recovery from severe deficits and a relatively low medication dose might have contributed to the relatively diminished effectiveness of escitalopram. Given higher baseline MADRS scores (mean of 16) in the Baseline-Blue group, a higher dose of escitalopram ( $>10 \mathrm{mg} /$ day) might have been necessary to reduce PSD risk at 3 months.

Consistent with a previous report, ${ }_{1}^{17}$ baseline neurological disability, as assessed by the NIHSS, was a significant predictor of PSD in all participants in our study. However, the degree of neurologic improvement (changes in NIHSS scores) during the first month was not an important determinant of PSD levels at 3 months, although there was a trend relationship between neurologic improvement and the decreased risk of PSD (odds ratio of 0.88 according to one-point improvement of NIHSS score). In a previous report from our group, we reported that changes in NIHSS scores during an initial 3 months of treatment were closely associated with changes in MADRS scores in patients with baseline depression (baseline MADRS $\geq 16$ [ $r=0.206, P=0.040]$ or a baseline MADRS score of 8 to 15 [ $r=0.171, P=0.049]$ ), but not in those without baseline depression (baseline MADRS $<8[r=$ $-0.023, P=0.76]$ ). ${ }^{11}$ Based on these findings, we speculated that patients with severe baseline depression and severe neurological deficits largely reflect a form of "reactive depression" that improves over time along with neurological recovery, masking the efficacy of escitalopram. In this post hoc analysis, however, changes in NIHSS were not clearly identified as an independent risk factor for PSD in patients with baseline depression. Different time periods employed in our current and earlier studies may have affected the diverging results. In this study, we used a shorter time period of 1 month after the index stroke (rather than 3 months) to evaluate the predictive effect of NIHSS score change on the development of PSD at 3 months. 
Table 5. Group-based differences in the clinical characteristics of patients with post-stroke depressive symptoms 3 months following stroke

\begin{tabular}{|c|c|c|c|}
\hline \multirow{2}{*}{ Characteristic } & \multicolumn{2}{|c|}{ PSD at 3 months } & \multirow{2}{*}{$P$} \\
\hline & Baseline-Pink ( $n=24)$ & Baseline-Blue ( $n=158)$ & \\
\hline \multicolumn{4}{|l|}{ Demographics } \\
\hline Age $(\mathrm{yr})$ & $62(51-78)$ & $69(59-76)$ & 0.344 \\
\hline Female sex & $10(41.7)$ & $70(44.3)$ & 0.808 \\
\hline \multicolumn{4}{|l|}{ Lesion location } \\
\hline Anterior cortex & $6(25.0)$ & $48(30.4)$ & 0.591 \\
\hline Thalamus & $1(4.2)$ & $13(8.2)$ & 0.698 \\
\hline Medulla & $1(4.2)$ & $13(8.2)$ & 0.698 \\
\hline Cerebellum & $1(4.2)$ & $16(10.1)$ & 0.704 \\
\hline Posterior cortex & $1(4.2)$ & $10(6.3)$ & $>0.999$ \\
\hline Subcortex & $14(58.3)$ & $77(48.7)$ & 0.381 \\
\hline Pons+Midbrain & $6(25.0)$ & $39(24.7)$ & $>0.999$ \\
\hline \multicolumn{4}{|l|}{ Clinical variables at baseline } \\
\hline MADRS & $4(2-6)$ & $18(12-24)$ & $<0.001$ \\
\hline NIHSS score & $4(3-7)$ & $5(3-7)$ & 0.831 \\
\hline Emotional incontinence & $1(4.2)$ & $10(6.3)$ & $>0.999$ \\
\hline Anger score & $24(19-26)$ & $24(21-28)$ & 0.280 \\
\hline MoCA score & $23(15-27)$ & $16(11-21)$ & 0.002 \\
\hline \multicolumn{4}{|c|}{ Clinical variables at 3 months } \\
\hline MADRS & $11(9-14)$ & $14(11-20)$ & 0.004 \\
\hline NIHSS score & $3(1-5)$ & $3(1-6)$ & 0.265 \\
\hline Emotional incontinence & $6(25.0)$ & $14(8.9)$ & 0.030 \\
\hline Anger score & $23(18-26)$ & $23(20-26)$ & 0.517 \\
\hline MoCA score & $27(18-28)$ & $19(11-23)$ & $<0.001$ \\
\hline
\end{tabular}

Values are presented as median (interquartile range) or number (\%).

PSD, post-stroke depression; MADRS, Montgomery-Asberg Depression Rating Scale; NIHSS, National Institute of Health Stroke Scale; MoCA, Montreal Cognitive Assessment.

We found that baseline cognitive dysfunction, measured by MoCA, was associated with PSD at 3 months across all patients (Table 4). Cognitive dysfunction, as measured by tools such as Mini-Mental State examination ${ }^{18}$ and Abbreviated Memory Test, ${ }^{19}$ has been shown to be associated with PSD. ${ }^{8}$ However, we also found that baseline cognitive dysfunction, which was more common in the Baseline-Blue group, was a significant predictor for PSD only among Baseline-Blue patients and not among Baseline-Pink patients (Table 4). In addition, among patients with PSD at 3 months, cognitive function was worse in the Baseline-Blue group than in the Baseline-Pink group (Table 5). The association between cognitive decline and persistent depression may, in part, be related to shared diagnostic items such as "difficulty in concentration." Alternatively, both depression and cognitive dysfunction may be common manifestations of damage in certain structures such as the frontal lobes or multiple small vessels which cause white matter changes. Unfortunately, this study was not able to explore the impact of small vessel disease on depression and cognitive dysfunction.

Generally, females are known to have a higher risk of PSD than males. ${ }^{20}$ Less social support after the index stroke may result in this outcome, considering that female patients are reported to receive relatively little support from both health care providers and the broader community. ${ }^{21,22}$ In our study, being female was not an independent predictor of PSD in all of the patients. However, being female increased the risk of PSD in the Baseline-Pink group but not in the Baseline-Blue group. The reason for this remains elusive. We speculate that, in the Baseline-Blue group there were other strong predictors (e.g., more severe neurologic deficits and cognitive dysfunction) of PSD and thus the contribution of sex to PSD might have been masked by our use of a multiple variable model.

Our results suggest that different approaches are necessary to treat/prevent PSD based on the presence of baseline depression. In patients without baseline depression, depressive symptoms gradually worsened over time if a placebo was given but 
improved with escitalopram (Figure 2). Escitalopram appears to be effective in this group of patients (Table 4) and therefore active pharmacological prevention might be a reasonable approach, especially in female patients or those with a mild stroke. Our findings align with a recent meta-analysis that reported on the efficacy of selective serotonin reuptake inhibitors (SSRIs) in preventing depression among initially non-depressed stroke patients. ${ }^{23}$ However, given a low rate of PSD development at 3 months (approximately 18\%) in the placebo users of the Baseline-Pink group, the cost-effectiveness and feasibility of this approach needs to be further evaluated. In contrast, in the Baseline-Blue group, escitalopram was found to be less effective and PSD was more closely related to neurological improvements. Given this, early and active rehabilitation may be necessary to promote the improvement of neurologic deficits and consequently of depressive symptoms in this group of patients. However, it should be further noted that, we used fixed doses $(10 \mathrm{mg}$ ) of escitalopram for a set duration (3 months). Therefore, it remains to be studied whether a higher dose and/ or longer duration of escitalopram administration, ${ }_{1}^{24}$ or the use of another class of antidepressant, would be more effective in treating Baseline-Blue patients.

Finally, among patients with PSD at 3 months, patients without baseline depression were more likely to have newly-developed emotional incontinence as compared to those with baseline depression (Table 5). It has been reported that emotional incontinence was closely associated with damages to subcortical structures (e.g., the basal ganglia or internal capsule). ${ }^{2,25}$ Because there are abundant serotonergic fibers in these areas $_{1}{ }^{26}$ altered neurotransmission after ischemic brain damage may play a role in the development of emotional incontinence. ${ }^{9}$ The more frequent development of emotional incontinence and relatively good response rates to escitalopram suggest that depressive symptoms in the Baseline-Pink group may be more closely associated with neurochemical changes ${ }^{27}$ due to brain damage than those in the Baseline-Blue group.

There are a number of limitations in the present study. First, we utilized post hoc analyses data from a randomized controlled trial. Therefore, baseline variables could not be balanced with the efficacy of escitalopram in each study group. Second, all patients were ethnically Korean, and the generalizability of our results to other ethnic populations may be limited. Third, in this clinical trial, we did not compile information on items such as patient socioeconomics, job status, or familial/social support, which may play roles in the development of depression. ${ }^{27}$ Finally, we examined the efficacy of escitalopram with a fixed dosage (10 mg/day) for a limited period.

Despite these limitations, our results demonstrate that pa- tient responses to escitalopram and predictors of PSD at 3 months may vary according to the presence of depression immediately following stroke. These findings highlight the heterogeneity of PSD pathophysiology and suggest the requirement for tailored therapeutic approaches in stroke patients who are at risk of PSD.

\section{Supplementary materials}

Supplementary materials related to this article can be found online at https://doi.org/10.5853/jos.2017.02712.

\section{Disclosure}

Jong S. Kim has received grants from Dong-A Pharmaceutical Company and from the Ministry for Health, Welfare, and Family Affairs, South Korea. All other authors declare no competing interests.

\section{Acknowledgments}

This study was supported by the Ministry for Health, Welfare, and Family Affairs, Republic of Korea (HI14C1985).

\section{References}

1. Robinson RG, Jorge RE. Post-stroke depression: a review. $A m$ J Psychiatry 2016;173:221-231.

2. Kim JS, Choi-Kwon S. Poststroke depression and emotional incontinence: correlation with lesion location. Neurology 2000;54:1805-1810.

3. Parikh RM, Robinson RG, Lipsey JR, Starkstein SE, Fedoroff JP, Price TR. The impact of poststroke depression on recovery in activities of daily living over a 2-year follow-up. Arch Neurol 1990;47:785-789.

4. House $A$, Dennis M, Mogridge $L$, Warlow $C$, Hawton $K$, Jones L. Mood disorders in the year after first stroke. Br $J$ Psychiatry 1991;158:83-92.

5. Wade DT, Legh-Smith J, Hewer RA. Depressed mood after stroke. A community study of its frequency. Br J Psychiatry 1987;151:200-205.

6. Aström $M$, Adolfsson $\mathrm{R}$, Asplund K. Major depression in stroke patients. A 3-year longitudinal study. Stroke 1993;24:976982.

7. Farner L, Wagle J, Engedal K, Flekkøy KM, Wyller TB, Fure B. Depressive symptoms in stroke patients: a 13 month followup study of patients referred to a rehabilitation unit. J Affect Disord 2010;127:211-218. 
8. Ayerbe L, Ayis $S$, Rudd AG, Heuschmann PU, Wolfe CD. Natural history, predictors, and associations of depression 5 years after stroke: the South London Stroke Register. Stroke 2011;42: 1907-1911.

9. Kim JS. Post-stroke mood and emotional disturbances: pharmacological therapy based on mechanisms. J Stroke 2016;18: 244-255.

10. Naess $H$, Kurtz M, Thomassen L, Waje-Andreassen U. Serial NIHSS scores in patients with acute cerebral infarction. Acta Neurol Scand 2016;133:415-420.

11. Kim JS, Lee EJ, Chang DI, Park JH, Ahn SH, Cha JK, et al. Efficacy of early administration of escitalopram on depressive and emotional symptoms and neurological dysfunction after stroke: a multicentre, double-blind, randomised, placebocontrolled study. Lancet Psychiatry 2017;4:33-41.

12. Kearns NP, Cruickshank CA, McGuigan KJ, Riley SA, Shaw SP, Snaith RP. A comparison of depression rating scales. Br J Psychiatry 1982;141:45-49.

13. Kim JS, Choi S, Kwon SU, Seo YS. Inability to control anger or aggression after stroke. Neurology 2002;58:1106-1108.

14. Brott $T$, Adams HP Jr, Olinger CP, Marler JR, Barsan WG, Biller $J$, et al. Measurements of acute cerebral infarction: a clinical examination scale. Stroke 1989;20:864-870.

15. Nasreddine ZS, Phillips NA, Bédirian V, Charbonneau $S$, Whitehead V, Collin I, et al. The Montreal Cognitive Assessment, MoCA: a brief screening tool for mild cognitive impairment. J Am Geriatr Soc 2005;53:695-699.

16. Higgins SL, Hummel JD, Niazi IK, Giudici MC, Worley SJ, Saxon $L A$, et al. Cardiac resynchronization therapy for the treatment of heart failure in patients with intraventricular conduction delay and malignant ventricular tachyarrhythmias. $J$ Am Coll Cardiol 2003:42:1454-1459.

17. Kutlubaev MA, Hackett ML. Part II: predictors of depression after stroke and impact of depression on stroke outcome: an updated systematic review of observational studies. Int $J$
Stroke 2014;9:1026-1036.

18. Tombaugh TN, McIntyre NJ. The mini-mental state examination: a comprehensive review. J Am Geriatr Soc 1992;40:922935.

19. Jitapunkul S, Pillay I, Ebrahim S. The abbreviated mental test: its use and validity. Age Ageing 1991;20:332-336.

20. Poynter B, Shuman M, Diaz-Granados N, Kapral M, Grace SL, Stewart DE. Sex differences in the prevalence of post-stroke depression: a systematic review. Psychosomatics 2009;50:563569.

21. Reeves MJ, Bushnell CD, Howard G, Gargano JW, Duncan PW, Lynch $G$, et al. Sex differences in stroke: epidemiology, clinical presentation, medical care, and outcomes. Lancet Neurol 2008;7:915-926.

22. Glader EL, Stegmayr B, Norving B, Terént A, Hulter-Asberg K, Wester PO, et al. Sex differences in management and outcome after stroke: a Swedish national perspective. Stroke 2003;34:1970-1975.

23. Salter KL, Foley NC, Zhu L, Jutai JW, Teasell RW. Prevention of poststroke depression: does prophylactic pharmacotherapy work? J Stroke Cerebrovasc Dis 2013;22:1243-1251.

24. Bech $P$, Andersen HF, Wade A. Effective dose of escitalopram in moderate versus severe DSM-IV major depression. Pharmacopsychiatry 2006;39:128-134.

25. Poeck K. Pathophysiology of emotional disorders associated with brain damage. In: Vinken PJ, Bruyn GW. Handbook of Clinical Neurology. New York, NY: Elsevier, 1969:343-367.

26. Kim JS. Post-stroke emotional incontinence after small lenticulocapsular stroke: correlation with lesion location. J Neurol 2002;249:805-810.

27. Choi-Kwon $S$, Han $K$, Choi $S$, Suh $M$, Kim YJ, Song $H$, et al. Poststroke depression and emotional incontinence: factors related to acute and subacute stages. Neurology 2012;78:11301137. 
Supplementary Table 1. Baseline characteristics of patients (placebo vs. escitalopram)

\begin{tabular}{|c|c|c|c|c|c|c|}
\hline \multirow{2}{*}{ Characteristic } & \multicolumn{3}{|c|}{ Baseline-Pink } & \multicolumn{3}{|c|}{ Baseline-Blue } \\
\hline & Placebo $(n=109)$ & Escitalopram $(n=94)$ & $P$ & Placebo $(n=128)$ & Escitalopram $(n=147)$ & $P$ \\
\hline \multicolumn{7}{|l|}{ At baseline } \\
\hline Age (yr) & $63.0 \pm 12.5$ & $61.7 \pm 11.8$ & 0.452 & $64.9 \pm 11.3$ & $65.4 \pm 12.7$ & 0.746 \\
\hline Female sex & $32(29.4)$ & 31 (33.0) & 0.578 & $54(42.2)$ & 70 (47.6) & 0.367 \\
\hline Hypertension & $80(73.4)$ & 76 (80.9) & 0.209 & 97 (75.8) & 107 (72.8) & 0.572 \\
\hline Diabetes & $47(43.1)$ & 31 (33.0) & 0.139 & $55(43.0)$ & $71(48.3)$ & 0.376 \\
\hline Hyperlipidemia & $57(52.3)$ & $41(43.6)$ & 0.217 & $62(48.4)$ & 75 (51.0) & 0.669 \\
\hline Coronary artery disease & $12(11.0)$ & $12(12.8)$ & 0.699 & $18(14.1)$ & $21(14.3)$ & 0.958 \\
\hline Smoking & $59(54.1)$ & $53(56.4)$ & 0.747 & $51(39.8)$ & $67(45.6)$ & 0.338 \\
\hline Lesion side & & & 0.097 & & & 0.739 \\
\hline Right & $55(50.5)$ & $40(42.6)$ & & $70(54.7)$ & $74(50.3)$ & \\
\hline Left & $45(41.3)$ & $51(54.3)$ & & $49(38.3)$ & $63(42.9)$ & \\
\hline Both & $9(8.3)$ & $3(3.2)$ & & $9(7.0)$ & $10(6.8)$ & \\
\hline \multicolumn{7}{|l|}{ Clinical variables } \\
\hline MADRS & $3.6 \pm 2.3$ & $3.2 \pm 2.5$ & 0.320 & $16.6 \pm 6.7$ & $15.5 \pm 6.7$ & 0.222 \\
\hline NIHSS & $4.2 \pm 2.6$ & $4.2 \pm 2.1$ & 0.922 & $5.2 \pm 3.0$ & $5.3 \pm 3.2$ & 0.686 \\
\hline Emotional incontinence & $6(5.5)$ & 0 & 0.021 & $12(9.4)$ & $14(9.5)$ & 0.966 \\
\hline Anger proneness & $22.9 \pm 5.1$ & $22.1 \pm 5.7$ & 0.278 & $23.2 \pm 5.6$ & $22.6 \pm 6.1$ & 0.405 \\
\hline MoCA & $20.0 \pm 6.4$ & $19.6 \pm 6.6$ & 0.656 & $18.1 \pm 6.9$ & $16.8 \pm 7.2$ & 0.145 \\
\hline
\end{tabular}

Values are presented as mean \pm standard deviation or number (\%).

MADRS, Montgomery-Asberg Depression Rating Scale; NIHSS, National Institute of Health Stroke Scale; MoCA, Montreal Cognitive Assessment. 
Supplementary Table 2. Risk factors of post-stroke depressive symptoms at 3 months by the presence of baseline depression

\begin{tabular}{|c|c|c|c|}
\hline \multirow{2}{*}{ Variable } & \multicolumn{2}{|c|}{ Crude odds ratio $(95 \% \mathrm{Cl})$} & \multirow{2}{*}{$\begin{array}{l}P \text { for interaction with } \\
\text { baseline depression }\end{array}$} \\
\hline & Baseline-Pink ( $n=296$ ) & Baseline-Blue ( $n=182)$ & \\
\hline \multicolumn{4}{|l|}{ Demographic and risk factors } \\
\hline Age $(/ 10 \mathrm{yr})$ & $1.01(0.97-1.05)$ & $1.03(1.01-1.05)^{*}$ & 0.372 \\
\hline Female sex & $1.70(0.71-4.06)$ & $0.93(0.57-1.50)$ & 0.235 \\
\hline Hypertension & $0.89(0.33-2.39)$ & $1.34(0.78-2.31)$ & 0.479 \\
\hline Diabetes & $0.78(0.32-1.92)$ & $1.58(0.98-2.57)$ & 0.174 \\
\hline Hyperlipidemia & $1.08(0.46-2.54)$ & $1.64(1.01-2.66)^{+}$ & 0.403 \\
\hline Coronary artery disease & $0.30(0.04-2.29)$ & $1.22(0.61-2.44)$ & 0.199 \\
\hline Smoking & $1.16(0.49-2.74)$ & $1.22(0.75-1.97)$ & 0.923 \\
\hline Lesion side & & & 0.879 \\
\hline Left vs. Right & $0.99(0.41-2.40)$ & $0.82(0.50-1.36)$ & \\
\hline Both vs. Right & $1.53(0.30-7.90)$ & $2.00(0.68-5.85)$ & \\
\hline \multicolumn{4}{|l|}{ Lesion location } \\
\hline Anterior cortex & $1.16(0.43-3.11)$ & $1.16(0.68-1.97)$ & 0.999 \\
\hline Thalamus & $0.35(0.04-2.70)$ & $1.08(0.44-2.61)$ & 0.320 \\
\hline Medulla & $0.48(0.06-3.77)$ & $0.96(0.41-2.27)$ & 0.540 \\
\hline Cerebellum & $0.61(0.08-4.87)$ & $1.54(0.63-3.72)$ & 0.421 \\
\hline Posterior cortex & $1.25(0.14-10.88)$ & $1.06(0.39-2.88)$ & 0.891 \\
\hline Subcortex & $1.66(0.70-3.93)$ & $1.15(0.71-1.85)$ & 0.467 \\
\hline Pons+Midbrain & $1.24(0.46-3.33)$ & $1.34(0.75-2.40)$ & 0.892 \\
\hline \multicolumn{4}{|l|}{ Clinical variables at baseline } \\
\hline Non-use of escitalopram (10 mg/day) & $5.00(1.67-14.29)^{*}$ & $1.56(0.96-2.56)$ & 0.058 \\
\hline MADRS & $1.19(0.98-1.44)$ & $1.16(1.11-1.22)^{*}$ & 0.791 \\
\hline NIHSS score & $1.25(1.05-1.48)^{+}$ & $1.07(0.99-1.16)$ & 0.117 \\
\hline Changes in NIHSS (0-4 wk) & $1.12(0.86-1.46)$ & $0.86(0.73-1.00)^{+}$ & 0.082 \\
\hline Emotional incontinence & $1.51(0.17-13.53)$ & $0.43(0.19-0.98)^{+}$ & 0.289 \\
\hline Anger score & $1.00(0.92-1.08)$ & $1.09(1.04-1.14)^{*}$ & 0.053 \\
\hline MoCA score & $1.02(0.95-1.09)$ & $0.92(0.88-0.95)^{*}$ & 0.008 \\
\hline
\end{tabular}

$\mathrm{Cl}$, confidence interval; MADRS, Montgomery-Asberg Depression Rating Scale; NIHSS, National Institute of Health Stroke Scale; MoCA, Montreal Cognitive Assessment.

${ }^{*} P<0.01 ;{ }^{+} P<0.05$. 